Original Article

\title{
Seasonality of restless legs syndrome: symptom variability in winter and summer times
}

\author{
Claudio Liguori ${ }^{\text {a, b, * }}$, Evi Holzknecht ${ }^{c}$, Fabio Placidi ${ }^{\text {a, b }}$, Francesca Izzi ${ }^{\text {a, b }}$, \\ Nicola Biagio Mercuri ${ }^{\text {b, d }}{ }^{\text {, Birgit Högl }}{ }^{c}$, Ambra Stefani ${ }^{c}$ \\ a Sleep Medicine Centre, Department of Systems Medicine, University of Rome Tor Vergata, Rome, Italy \\ ${ }^{\mathrm{b}}$ UOC Neurologia, University Hospital of Rome Tor Vergata, Rome, Italy \\ ${ }^{\mathrm{c}}$ Department of Neurology, Medical University of Innsbruck, Innsbruck, Austria \\ d IRCCS Fondazione Santa Lucia, Rome, Italy
}

\section{A R T I C L E I N F O}

\section{Article history:}

Received 24 May 2019

Received in revised form

14 July 2019

Accepted 29 July 2019

Available online 14 August 2019

\section{Keywords:}

Restless legs syndrome

Seasonality

Summer

Winter

IRLS

ESS

\begin{abstract}
A B S T R A C T
Introduction: Restless legs syndrome (RLS) is a common sensorimotor neurological disorder, with symptoms that might cause sleep fragmentation leading to excessive daytime sleepiness. A seasonality of RLS symptoms has been suggested; however, to date, no study focused on this aspect. In order to detect a possible seasonality of RLS manifestations, we evaluated RLS symptom severity and excessive daytime sleepiness in winter and summer in RLS patients.

Methods: RLS patients who performed two follow-up visits in summer and winter were included in this retrospective bicentric analysis. RLS severity, measured with the International RLS Study Group rating scale (IRLS), and daytime sleepiness, measured with the Epworth Sleepiness Scale (ESS), were recorded in both seasons in Innsbruck and Rome Sleep Medicine Centers.

Results: In sum, 64 RLS patients were included. In the overall sample, IRLS in summer was higher than in winter $(p=0.008)$. After gender stratification, this held true only in men $(p=0.008)$. When stratifying for centers, the seasonal variation in RLS severity was present exclusively in Rome $(p<0.001)$. Moreover, 20 RLS patients completed ESS in both seasonal periods, and scores in summer were higher than in winter $(\mathrm{p}<0.001)$.

Conclusion: This retrospective observational study showed an increase of RLS severity during summer compared to winter, supporting the hypothesis that RLS symptoms are more troublesome when temperatures are higher. Changes in microvascular regulation, sweating, and serum iron level changes may support this difference in RLS symptoms across the year. The documented seasonal variation in RLS severity with worsening in the warmer months needs to be investigated further in prospective studies.
\end{abstract}

() 2019 Elsevier B.V. All rights reserved.

\section{Introduction}

Restless legs syndrome (RLS) is a neurological sleep disorder, more frequently affecting women than men, with a prevalence up to $11 \%$ in the general population [1-4]. RLS has been associated with considerable impairment in quality of life, as it can cause sleep fragmentation with consequent daytime sleepiness, mood disturbances or reduced concentration, among others [5,6]. The most widely accepted pathophysiological mechanisms of RLS include

\footnotetext{
* Corresponding author. Sleep Medicine Centre, Department of Systems Medicine, University of Rome "Tor Vergata", Viale Oxford 81, 00133, Rome, Italy. Fax: +39 0620902116.
}

E-mail address: dott.claudioliguori@yahoo.it (C. Liguori). genetic predisposition, central nervous system iron dysregulation, and dopaminergic dysfunction [7]. Peripheral hypoxia has also been reported to play a role in RLS, and a possible pathophysiologic link between peripheral hypoxia and RLS symptoms has been suggested [8-10].

Seasonality in sleep disorders has been hypothesized in the past. In particular, light exposure, climate changes, and daytime length have been discussed as possible determinants of sleep disorders [11]. A seasonal variation in RLS symptomatology has also been previously suggested [11]. However, scarce and controversial evidence is present in the literature. Evidence of seasonal trends in RLS has been exclusively drawn from Internet search query data [12]. Moreover, despite some clinical observations, seasonal variations in RLS symptom severity have not been systematically evaluated. 
On this basis, the present study aimed to systematically investigate RLS symptom severity measured by the International Restless Legs Syndrome Study Group (IRLSSG) rating scale (IRLS) in consecutive summer and winter periods in RLS patients, in order to evaluate possible changes in RLS symptoms between the hot and cold seasons. Moreover, since RLS symptoms may impair night sleep and therefore cause daytime sleepiness, we also measured excessive daytime sleepiness using the Epworth Sleepiness Scale (ESS).

\section{Methods}

This is a retrospective, observational study based on individual chart review of patients affected by primary RLS. Patients were classified as affected by RLS according to IRLSSG criteria [1]. Data were collected at the two centers participating in the study, the Sleep Medicine Centre of the University Hospital of Rome "Tor Vergata" and the Sleep Laboratory of the Department of Neurology, Medical University of Innsbruck. Eligible patients were retrospectively selected among those who were followed regularly by the two centers and underwent clinical follow-up in two consecutive time points: the winter period and the summer period. Considering the different meteorological features of Innsbruck, Austria and Rome, Italy, we choose the hottest and coldest periods in these two geographical areas according to the data of the World Meteorological Organization. In particular, the winter period was considered from December to January in Innsbruck [mean daily temperature $(-1)-(-1.7)^{\circ} \mathrm{C}$ ] and from January to February in Rome [mean daily temperature $(7.5)-(8.2)^{\circ} \mathrm{C}$ ], and the summer period was considered from June to July in Innsbruck [mean daily temperature (16.1) $-(18.1)^{\circ} \mathrm{C}$ ] and from July to August in Rome [mean daily temperature $\left.(24.1)-(24.5)^{\circ} \mathrm{C}\right]$. We exclusively included patients without changes in their RLS treatment between the two periods and excluded those who presented augmentation, and who presented secondary RLS. Demographic data were collected, as well as clinical data, including age at RLS onset, current RLS treatment, IRLS, and ESS scores. The IRLS, previously provided by the IRLSSG [13], is a validated and widely used scale to quantify and monitor RLS symptoms and assess the severity of the disease. The ESS is a widely used instrument to assess daytime sleepiness and already validated also in RLS patients [14].

Based on the previous preliminary evidence about the increase of RLS symptoms in summer compared to winter [11,12], we planned to test in a pilot retrospective observational study whether there is a difference in the IRLS scores measured in summer and winter in patients affected by RLS under stable treatments. We also planned to perform a stratification for each center, as the two participating centers (Rome and Innsbruck) are at different latitudes and have a different climate. The statistical analysis was performed using SPSS version 24. Data distribution was assessed using the Kolmogorov-Smirnov test. As data were normally distributed, data are shown as mean and standard deviation. For group comparison, parametric tests were applied. Correlations were examined using Pearson's correlation coefficient. A p-value $<0.05$ was considered statistically significant.

The study was approved by the local Ethics Committees of the University Hospital of Rome "Tor Vergata" and the Medical University of Innsbruck.

\section{Results}

A total of 64 patients with RLS were included in this retrospective analysis. Of those, 35 RLS patients were recruited in Innsbruck, and 29 RLS patients were recruited in Rome. Demographic and clinical data are presented in Table 1.

\subsection{RLS symptom severity}

RLS symptom severity measured with the IRLS in summer and winter, and the difference in IRLS scores between summer and winter (delta IRLS), did not differ between patients from Innsbruck and Rome; this also was observed after stratification for gender and treatment (all p > 0.05). No gender differences were found in IRLS in summer, IRLS in winter, and delta IRLS, also after stratification for center and treatment (all $\mathrm{p}>0.05$ ).

In the overall sample, IRLS in summer was higher than in winter $(\mathrm{p}=0.008)$. Individual variations in IRLS between summer and winter are shown in Fig. 1. After gender stratification, the difference was significant only in men ( $\mathrm{p}=0.008$, Fig. 2$)$. When stratifying for centers, the difference in RLS severity was present exclusively in patients from Rome ( $p<0.001$, Fig. 3 ). These results did not change after gender stratification.

Considering the change of IRLS scores between summer and winter, we documented an increase of at least three and five points in $28 / 64(43.8 \%$ ) and $18 / 64$ (28.1\%) patients, respectively (Table 2). This increase was more evident in men (12/22 patients, 54.5\%), and in Rome (17/29 patients, 58.6\%) (Table 2).

When analyzing different treatment groups separately, IRLS in summer, IRLS in winter, and delta IRLS did not differ between patients with and without RLS therapy (all $\mathrm{p}>0.05$ ). Also, after stratification for each center, no differences were found (all $\mathrm{p}>0.05$ ). Moreover, in the whole sample, IRLS in summer was higher (ie, more severe symptoms) than in winter in treated patients, but not in untreated patients $(\mathrm{p}=0.002)$. Among treated patients, statistically relevant differences were present only in men after stratification for gender, and only in patients from Rome after stratification for center. Details are shown in Table 2.

A correlation between IRLS in summer and winter was present (Pearson's r 0.581, p $<0.001$ ).

\subsection{Daytime sleepiness}

Twenty out of 64 patients completed the ESS scores in both periods. ESS in summer was higher than ESS in winter in the overall sample $(\mathrm{p}<0.001)$. No gender differences, nor differences between centers or between treated and untreated patients were found (all $\mathrm{p}>0.05$ ). These data are shown in Table S1. ESS scores in summer and winter correlated strongly (Pearson's r 0.687, $\mathrm{p}=0.001$ ).

No significant correlation between IRLS and ESS in summer was found $(0.044, \mathrm{p}=0.82)$, as well as between IRLS and ESS in winter $(0.098, \mathrm{p}=0.667)$.

\section{Discussion}

The purpose of the present retrospective observational study was to investigate whether RLS symptoms may exhibit a seasonal variability, with a particular focus on the coldest and hottest periods of the year. Briefly, we used IRLS to quantify RLS symptom severity during summer and winter in RLS patients with stable RLS therapy during the study period. We documented a significant increase in IRLS scores during the summertime compared to winter, thus supporting the hypothesis that RLS symptoms are more troublesome when temperatures increase. Therefore, a seasonal variation in RLS symptoms seems to be present, with worsening in the warmer months. However, when stratifying for centers, the comparison between the two seasons was statistically significant exclusively in RLS patients followed in Rome, although RLS patients from Innsbruck showed a trend towards higher scores in summer. Moreover, after gender stratification, the difference persisted only in men. 
Table 1

Demographic and clinical data.

\begin{tabular}{|c|c|c|c|c|}
\hline & Overall sample $(\mathrm{n}=64)$ & Innsbruck $(\mathrm{n}=35)$ & Rome $(\mathrm{n}=29)$ & P value \\
\hline Age, years & $55.5 \pm 13.3$ & $61.8 \pm 15.3$ & $48.0 \pm 14.4$ & $<0.001$ \\
\hline Sex, women n (\%) & $42(65.6)$ & $24(68.6)$ & $18(62.1)$ & 0.608 \\
\hline \multicolumn{5}{|l|}{ Therapy: } \\
\hline No therapy, n (\%) & $10(15.5)$ & $7(20)$ & $3(10.3)$ & 0.327 \\
\hline Dopaminergic, n (\%) & $33(51.6)$ & $19(54.3)$ & $14(48.3)$ & 0.802 \\
\hline Other therapies, n (\%) & $21(32.8)$ & $9(25.7)$ & $12(41.1)$ & 0.285 \\
\hline
\end{tabular}

Data are presented as mean \pm standard deviation (standard error of the mean), and $\mathrm{n}(\%)$. Other therapies include non-dopaminergic treatment and polytherapy.

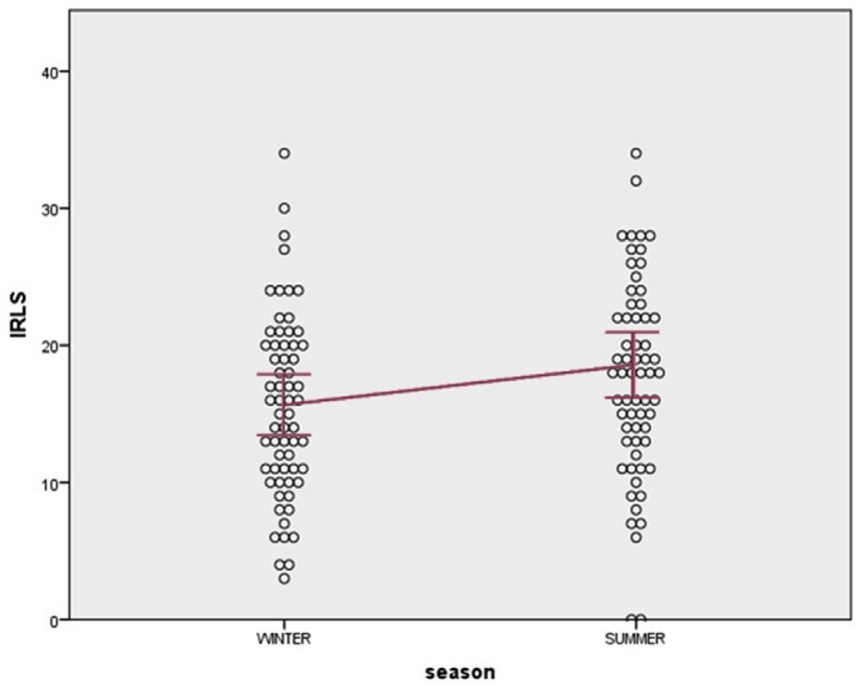

Fig. 1. IRLS in winter and summer. Box plot with the mean value \pm standard deviation of the IRLS in winter and summer in the whole population. IRLS, international Restless Legs Syndrome severity scale.

Although previous case reports hypothesized a seasonal pattern in RLS [11,12], to date it had not been analyzed if RLS symptoms present variations during the year. Only one previous study tried to

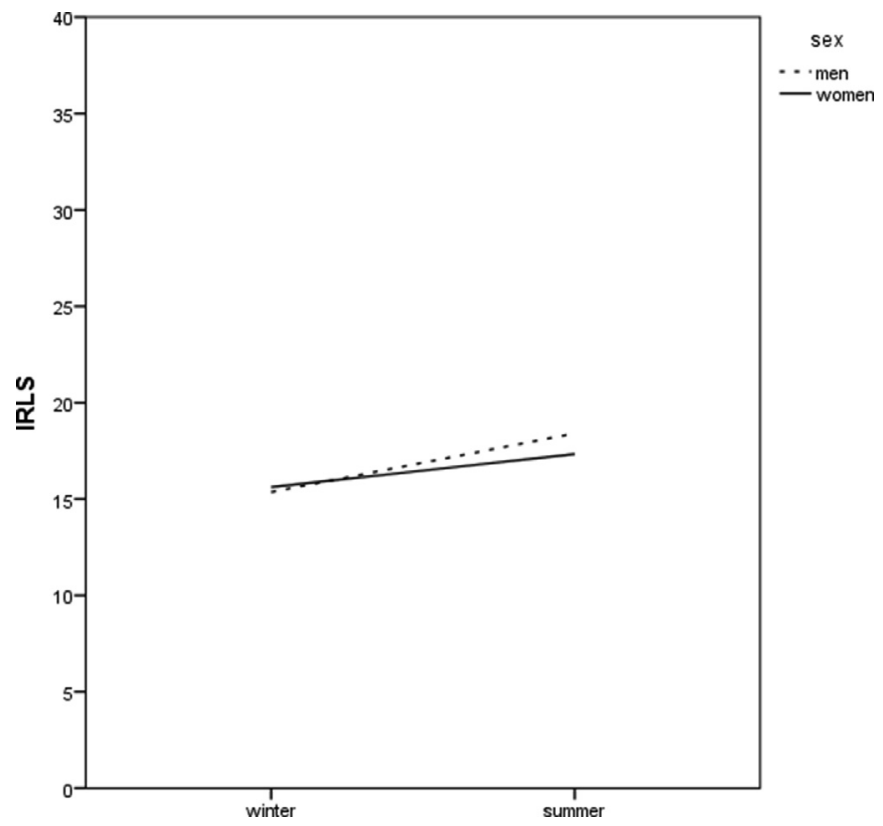

Fig. 2. IRLS in winter and summer in men and women. Mean value of the IRLS in winter and summer in men and women is shown. IRLS, international Restless Legs Syndrome severity scale. examine this phenomenon by analyzing Internet search query data about RLS symptomatology, and documented a seasonal trend of the search queries, with a peak in the summer months [12]. However, the previously observed seasonality of web searches for RLS symptoms and leg cramps has been re-examined later on, and that author did not find the same seasonal effect for RLS searches in Internet data, although he did not exclude a clinical significance of the previously identified seasonality of RLS symptoms [15].

In the present study, we confirmed the evidence of seasonality in RLS, showing that patients affected by RLS presented higher IRLS scores during summer compared to winter. It is widely accepted that CNS iron deficiency plays a central role in the pathophysiology of RLS [16]. Serum iron levels measurably change across the year. In healthy volunteers, a decrease in serum ferritin, transferrin and iron concentration and an increase in soluble transferrin receptor during summer have been documented [17]. Patients affected by RLS frequently present a dysfunctional iron metabolism and a significant reduction in iron levels [18]; Thus, the reduced iron concentrations during the hottest months may contribute to more pronounced RLS symptoms during summer. A further explanation may be driven by the vascular hypothesis of RLS pathophysiology. The reduced regulation of microvascular circulation and the subsequently reduced heat tolerability during summer have been widely demonstrated $[19,20]$. In an experimental study it has been documented that, when exposed to temperature around $30{ }^{\circ} \mathrm{C}$ (easily reached in Rome in the hottest summer months, but rarely in Innsbruck) RLS patients present a more uniform temperature in the core body and lower limbs, compared to controls, in whom feet

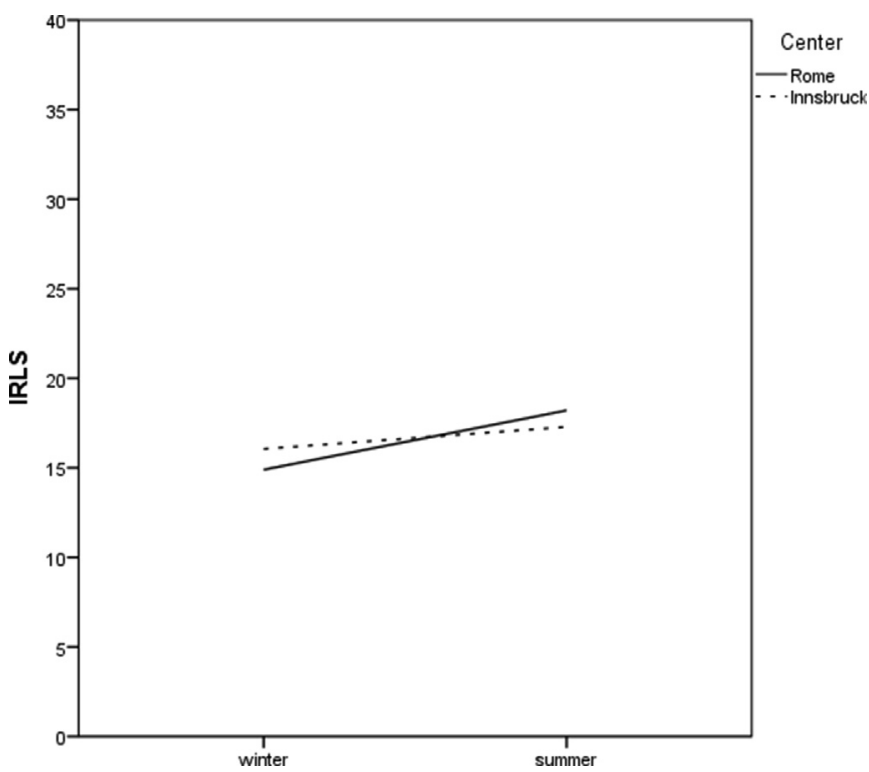

Fig. 3. IRLS in winter and summer in the two centres (Rome and Innsbruck). Mean value of the IRLS in winter and summer in Rome and Innsbruck is shown. IRLS, international Restless Legs Syndrome severity scale. 
Table 2

Comparison of RLS severity in summer and winter.

\begin{tabular}{|c|c|c|c|c|c|c|}
\hline & IRLS summer & IRLS winter & Delta IRLS & $\mathrm{P}$ & Delta IRLS $\geq 3, \mathrm{n}(\%)$ & Delta IRLS $\geq 5, \mathrm{n}(\%)$ \\
\hline Overall sample $(n=64)$ & $17.7 \pm 7.1$ & $15.5 \pm 6.7$ & $2.2 \pm 6.3$ & 0.008* & $28(43.8)$ & $18(28.1)$ \\
\hline $\operatorname{Men}(\mathrm{n}=22)$ & $18.4 \pm 7.3$ & $15.4 \pm 6.7$ & $3.1 \pm 4.9$ & $0.008^{*}$ & $12(54.5)$ & $6(27.3)$ \\
\hline Women $(\mathrm{n}=42)$ & $17.3 \pm 7.1$ & $15.6 \pm 6.5$ & $1.7 \pm 1.1$ & 0.117 & $16(38.1)$ & $12(28.6)$ \\
\hline Innsbruck $(\mathrm{n}=35)$ & $17.3 \pm 8.4$ & $16.1 \pm 7.9$ & $1.2 \pm 7.6$ & 0.347 & $11(31.4)$ & $10(28.6)$ \\
\hline $\operatorname{Men}(\mathrm{n}=11)$ & $19.4 \pm 9.2$ & $16.9 \pm 9.0$ & $2.4 \pm 5.8$ & 0.195 & $4(36.4)$ & $4(36.4)$ \\
\hline Women $(n=24)$ & $16.3 \pm 8.0$ & $15.7 \pm 7.0$ & $0.7 \pm 8.4$ & 0.700 & $7(29.2)$ & $6(25)$ \\
\hline Rome $(\mathrm{n}=29)$ & $18.2 \pm 5.4$ & $14.9 \pm 5.2$ & $3.3 \pm 4.1$ & $<0.001^{*}$ & $17(58.6)$ & $8(27.6)$ \\
\hline $\operatorname{Men}(n=11)$ & $17.4 \pm 5.2$ & $13.8 \pm 3.8$ & $3.6 \pm 3.9$ & 0.011* & $8(72.7)$ & $2(18.2)$ \\
\hline Women $(n=18)$ & $18.7 \pm 5.6$ & $15.6 \pm 5.9$ & $3.1 \pm 4.2$ & $0.006^{*}$ & $9(50)$ & $6(33.3)$ \\
\hline \multicolumn{7}{|l|}{ RLS treatment } \\
\hline Untreated $(\mathrm{n}=10)$ & $15.6 \pm 8.2$ & $14.3 \pm 7.7$ & $1.3 \pm 7.1$ & 0.579 & $4(40)$ & $4(40)$ \\
\hline Treated $(\mathrm{n}=54)$ & $18.1 \pm 6.9$ & $15.8 \pm 6.4$ & $2.3 \pm 6.2$ & $0.002^{*}$ & $24(44.4)$ & $14(25.9)$ \\
\hline $\operatorname{Men}(n=16)$ & $18.1 \pm 7.6$ & $15.2 \pm 6.8$ & $2.8 \pm 4.4$ & $0.022^{*}$ & $9(56.3)$ & $3(18.8)$ \\
\hline Women $(\mathrm{n}=38)$ & $18.1 \pm 6.7$ & $16.0 \pm 6.4$ & $2.1 \pm 6.9$ & 0.063 & $15(39.5)$ & $11(28.9)$ \\
\hline Rome $(n=26)$ & $18.5 \pm 5.6$ & $15.3 \pm 5.2$ & $3.1 \pm 4.0$ & $0.001^{*}$ & $15(57.7)$ & $6(23.1)$ \\
\hline Innsbruck $(\mathrm{n}=28)$ & $17.7 \pm 8.1$ & $16.2 \pm 7.5$ & $1.6 \pm 7.7$ & 0.289 & $9(32.1)$ & $8(28.6)$ \\
\hline
\end{tabular}

All data presented as mean \pm standard deviation. RLS, restless legs syndrome.

Significant p value set at $<0.05$; * statistical significant; in bold statistically significant results.

are cooler than the core body temperature. No difference between RLS patients and controls was present when exposed to low temperatures [21]. These findings suggest impaired spatial cooling at a higher temperature in RLS patients and further support the hypothesis that heat may increase RLS symptomatology due to the presence of an already impaired microvascular circulation. Coupled with microvascular changes, also autonomic nervous system (ANS) dysregulation is present in patients affected by RLS [22]. ANS actively controls sweating, among other functions [23]. Considering the altered autonomic innervation in RLS patients, in particular in the lower limbs, also sweating can be dysregulated in those patients (with excessive sweating in the trunk and reduced sweating in the limbs), thus not reducing the temperature in the lower limbs during the hottest season. In line with this hypothesis, after stratification for gender, we found a significant worsening of RLS symptoms in men, and after stratification for each center, the worsening in summer was evident in Rome. Men usually present increased sweating with a higher local and whole-body sudomotor activity compared to women as a result of increased body temperature [24-26]. Therefore, it might be hypothesized that dysregulated thermoregulation, coupled with the altered ANS responses in RLS patients, could explain the worsening of RLS symptoms in men during summer in Rome [18]. Although we can only suppose the pathophysiological basis of this finding, it is conceivable that hormonal changes and comorbid insomnia, which are all more frequent in women than in men, may play a major role in RLS pathogenesis in women. Furthermore, this gender difference may be related to iron deficiency, since men tend to have higher ferritin levels in adulthood compared to women [18]. Therefore, it is tempting to speculate that hormonal factors, insomnia, and iron deficiency play a major role in women, and as these factors are less influenced by seasonality, the worsening of symptoms in the hottest months in women is milder than in men. Sweating is associated with lower serum ferritin levels, as sweat contains an average 300-400 $\mu \mathrm{g} / \mathrm{l}$ iron, and during moderate exercise at temperature $>10^{\circ} \mathrm{C}$ sweat loss can reach $2-3 \mathrm{l} / \mathrm{h}$, with daily iron losses of 1-2 mg (approximately the amount introduced with the diet) [29]. Hence, it is possible to hypothesize that during the hottest months, serum ferritin levels may reduce in men more than in women, as sweating is more marked in men, therefore lowering iron levels and increasing RLS symptoms more markedly in men than in women during summer [30]. However, although a dysregulation of sweating in RLS may be suggested, this has not been investigated yet. Finally, the interpretation of our findings also needs to take into account a possible gender difference in self- perception of RLS symptoms, since women tend to report symptoms at a lower severity level compared with men [31]. Furthermore, both microvascular circulation dysregulation and ANS impairment may increase RLS symptoms during summer. Low doses of dopamine and dopamine agonists produce vasodilatation [27], already caused by heat during summer; these factors can increase RLS symptoms by altering temperature perception and increasing lower limbs temperature and swelling. Our findings that RLS symptoms severity worsened in summer only in treated patients support this hypothesis. However, further investigations are needed to confirm these entirely speculative hypotheses; also to be taken into account is the observation that not all patients affected by RLS present the same trend of an increase of IRLS scores during summer and a reduction during winter. This finding may also be related to the evidence that several mechanisms may contribute to the supposed seasonality of RLS symptoms. Moreover, several predisposing factors contribute to RLS pathogenesis, which can be considered a multifactorial disease, and these have different relevance in each patient. For example, genetic risk factors, which are more relevant for early-onset RLS, along with menopause, parity, and hormonal changes, may significantly change RLS symptoms in women [18]. Additionally, symptoms of RLS can be different, and patients with RLS may report different perceptions of their symptoms, as suggested by the numerous verbal descriptions of their troublesome and uncomfortable symptoms, such as tingling, pulling, stinging, burning, and crawling [28].

Thus, when separately analyzing the data of Rome and Innsbruck, a statistically significant difference in symptoms severity between summer and winter IRLS scores was present only in Rome. This difference can be ascribed to meteorological factors since summer in Rome is hotter and more humid than in Innsbruck. Consistently, this more pronounced increase in RLS symptoms observed in patients from Rome could be due to the higher temperatures reached in Rome (coupled with higher humidity) as compared to Innsbruck, aggravated by the fact that sweating may increase RLS symptoms by negatively changing iron and serum ferritin levels (as previously discussed).

We are aware that this study presents limitations intrinsic to the study design. The main limitations are the retrospective design, which might imply a bias in patient selection, and the relatively small sample of patients, although recruited by two centers located at different latitudes. Furthermore, we did not include patients with augmentation because of the study design (no changes in therapy allowed between the two evaluations). Finally, we did not have information about serum ferritin/iron levels for all patients included in the study. 
The strengths of this study are the inclusion of two centers at different latitudes, stable medications during the observational period, a systematic analysis in order to obtain data about the seasonality of RLS symptoms, and strict inclusion and exclusion criteria.

In conclusion, we report new, preliminary findings of seasonality in RLS symptomatology. Our results support the hypothesis that RLS symptoms' severity changes during the year, with a worsening during summer, which is more evident at latitudes where summer is definitively hot (as in Rome). Changes in microvascular regulation, sweating, and serum iron levels modifications may underlie this difference in RLS symptoms across the year. However, our conclusions should be considered as a preliminary impression, which requires further validation from prospective studies on larger populations, evaluating also the effect of seasonality on augmentation.

\section{Acknowledgments/Conflicts of interest/Funding sources for this study}

Claudio Liguori has been a consultant and/or attended the scientific advisory board for Eisai, MSD and Pfizer. Dr. Fabio Placidi received Research Support from EISAI Pharmaceuticals. Dr. Francesca Izzi has been a consultant and/or attended scientific advisory board for Eisai. Nicola Biagio Mercuri declares no conflict of interest or financial disclosures.

Birgit Högl reports speaker honoraria from Otsuka, UCB, Eli Lilly, Janssen Cilag, Lundbeck Abbvie, Nutricia, Inspire; consulting/advisory boards for Mundipharma, Benevolent Bio, Axovant, AoP Orphan, Roche; travel support from Habel Medizintechnik, Vivisol Austria. Ambra Stefani reports travel support from Habel Medizintechnik, OSG, UCB; support for research and consulting from Axovant. Evi Holzknecht declares no conflict of interest or financial disclosures.

We thank Luigi Mossuto for the graphical support.

We confirm that we have read the Journal's position on issues involved in ethical publication and affirm that this report is consistent with those guidelines.

\section{Authors' contributions}

Claudio Liguori, Ambra Stefani: design of the study.

Claudio Liguori, Ambra Stefani, Evi Holzknecht: data acquisition.

Claudio Liguori, Ambra Stefani: drafting the manuscript and interpretation of the data.

Evi Holzknecht: statistical analysis.

Francesca Izzi, Fabio Placidi and Birgit Högl: critical revision of the manuscript.

Nicola Biagio Mercuri, Birgit Högl, Fabio Placidi: study supervision.

\section{Conflict of interest}

The ICMJE Uniform Disclosure Form for Potential Conflicts of Interest associated with this article can be viewed by clicking on the following link: https://doi.org/10.1016/j.sleep.2019.07.026.

\section{Appendix A. Supplementary data}

Supplementary data to this article can be found online at https://doi.org/10.1016/j.sleep.2019.07.026.

\section{References}

[1] American academy of sleep medicine international classification of sleep disorders. 3rd ed. 2014 (ICSD-3).
[2] Högl B, Kiechl S, Willeit J, et al. Restless legs syndrome: a community-based study of prevalence, severity, and risk factors. Neurology 2005 Jun 14;64(11):1920-4.

[3] Winkelman JW, Bogan RK, Schmidt MH, et al. Randomized polysomnography study of gabapentin enacarbil in subjects with restless legs syndrome. Mov Disord 2011 Sep;26(11):2065-72.

[4] Allen RP, Bharmal M, Calloway M. Prevalence and disease burden of primary restless legs syndrome: results of a general population survey in the United States. Mov Disord 2011 Jan;26(1):114-20.

[5] Ferini-Strambi L, Walters AS, Sica D. The relationship among restless legs syndrome (Willis-Ekbom Disease), hypertension, cardiovascular disease, and cerebrovascular disease. J Neurol 2014 Jun;261(6):1051-68.

[6] Earley CJ, Silber MH. Restless legs syndrome: understanding its consequences and the need for better treatment. Sleep Med 2010 Oct;11(9):807-15.

[7] Guo S, Huang J, Jiang $\mathrm{H}$, et al. Restless legs syndrome: from pathophysiology to clinical diagnosis and management. Front Aging Neurosci 2017 Jun 2;9:171.

[8] Salminen AV, Rimpilä V, Polo O. Peripheral hypoxia in restless legs syndrome (Willis-Ekbom disease). Neurology 2014 May 27;82(21):1856-61.

[9] Patton SM, Ponnuru P, Snyder AM, et al. Hypoxia-inducible factor pathway activation in restless legs syndrome patients. Eur J Neurol 2011 Nov;18(11): 1329-35.

10] Stefani A, Heidbreder A, Hackner $\mathrm{H}$, et al. Influence of high altitude on periodic leg movements during sleep in individuals with restless legs syndrome and healthy controls: a pilot study. Sleep Med 2017 Jan;29:88-9.

[11] Zucconi M, Manconi M. Sleep disturbances in restless legs syndrome (RLS) and periodic limb movements (PLM). In: Pandi-Perumal SR, Monti JM, editors. Clinical pharmacology of sleep. Birkhäuser Basel; 2006.

[12] Ingram DG, Plante DT. Seasonal trends in restless legs symptomatology: evidence from Internet search query data. Sleep Med 2013 Dec;14(12): 1364-8.

[13] Allen RP, Picchietti DL, Garcia-Borreguero D, et al. Restless legs syndrome Willis-Ekbom disease diagnostic criteria: updated International Restless Legs Syndrome Study Group (IRLSSG) consensus criteria - history, rationale, description, and significance. Sleep Med 2014 Aug;15(8):860-73.

[14] Johns MW. A new method for measuring daytime sleepiness: the Epworth sleepiness scale. Sleep 1991 Dec;14(6):540-5.

[15] O'Keeffe ST. Summertime blues? A re-examination of the seasonality of web searches for restless legs and leg cramps. Sleep Med 2017 Sep;37:119-23.

[16] Allen RP, Earley CJ. The role of iron in restless legs syndrome. Mov Disord 2007:22(Suppl 18):S440-8.

[17] Maes M, Bosmans E, Scharpé S, et al. Components of biological variation in serum soluble transferrin receptor: relationships to serum iron, transferrin and ferritin concentrations, and immune and haematological variables. Scand J Clin Lab Investig 1997 Feb;57(1):31-41.

[18] Manconi M, Ulfberg J, Berger K, et al. When gender matters: restless legs syndrome. Report of the "RLS and woman" workshop endorsed by the European RLS Study Group. Sleep Med Rev 2012 Aug;16(4):297-307.

[19] Connor JR, Patton SM, Oexle K, et al. Iron and restless legs syndrome: treatment, genetics and pathophysiology. Sleep Med 2017 Mar;31:61-70.

[20] Nicolaides AN, Allegra C, Bergan J, et al. Management of chronic venous disorders of the lower limbs: guidelines according to scientific evidence. Int Angiol 2008 Feb;27(1):1-59.

[21] Anderson KN, Di Maria C, Allen J. Novel assessment of microvascular changes in idiopathic restless legs syndrome (Willis-Ekbom disease). J Sleep Res 2013 Jun;22(3):315-21.

[22] Rocchi C, Albanese M, Placidi F, et al. Chronic dopaminergic treatment in restless legs syndrome: does it affect the autonomic nervous system? Sleep Med 2015 Sep;16(9):1071-6.

[23] Minota K, Coon EA, Benarroch EE. Neurologic aspects of sweating and its disorders. Neurology 2019 Apr 24. https://doi.org/10.1212/WNL.00000 00000007540 [Epub ahead of print].

[24] Gagnon D, Crandall CG, Kenny GP. Sex differences in postsynaptic sweating and cutaneous vasodilation. J Appl Physiol (1985) 2013 Feb;114(3):394-401.

[25] Gagnon D, Kenny GP. Sex differences in thermoeffector responses during exercise at fixed requirements for heat loss. J Appl Physiol (1985) 2012 Sep $1 ; 113(5): 746-57$.

[26] Gagnon D, Kenny GP. Sex modulates whole-body sudomotor thermosensitivity during exercise. J Physiol 2011 Dec 15:589(Pt 24):6205-17.

[27] Kujawa K, Leurgans S, Raman R, et al. Acute orthostatic hypotension when starting dopamine agonists in Parkinson's disease. Arch Neurol 2000 Oct:57(10):1461-3.

[28] Karroum EG, Golmard JL, Leu-Semenescu S, et al. Sensations in restless legs syndrome. Sleep Med 2012 Apr;13(4):402-8.

[29] Chatard JC, Mujika I, Guy C, et al. Anaemia and iron deficiency in athletes. Practical recommendations for treatment. Sports Med 1999 Apr;27(4): 229-40.

[30] Casoni I, Borsetto C, Cavicchi A, et al. Reduced hemoglobin concentration and red cell hemoglobinization in Italian marathon and ultramarathon runners. Int J Sports Med 1985 Jun;6(3):176-9.

[31] Yeh P, Walters AS, Tsuang JW. Restless legs syndrome: a comprehensive overview on its epidemiology, risk factors, and treatment. Sleep Breath 2012 Dec;16(4):987-1007. 